\title{
Changes in microplanktonic protists assemblages promoted by the thermocline induced stratification around an oceanic archipelago
}

\author{
ALEJANDRO E.S.F. DA COSTA ${ }^{1}$, JANA R. DE SANTANA ${ }^{1,2}$ and SIGRID NEUMANN-LEITÃO ${ }^{1}$ \\ ${ }^{1}$ Laboratório de Zooplâncton, Museu de Oceanografia Prof. Petrônio Alves Coelho, Universidade \\ Federal de Pernambuco, Avenida da Arquitetura, s/n, 50740-550 Recife, PE, Brazil \\ ${ }^{2}$ Departamento de Ciências Humanas e Tecnologias, Universidade do Estado da Bahia, \\ Rua Professor Carlos Santos, 601, 47400-000 Xique-Xique, BA, Brazil
}

Manuscript received on August 4, 2017; accepted for publication on January 23, 2018

\begin{abstract}
In the area around the archipelago of São Pedro e São Paulo (ASPSP), a set of small rocky islands in the Equatorial Atlantic, the thermocline begins at a more superficial depth than in the surrounding waters. This raises the question if there is any change in heterotrophic microplanktonic protists assemblages occurring in the surface mixed layer and in the subsurface layer. To answer this question, we sampled seawater in seven depths (between 1-100 m) with a 10L Niskin bottle and obtained temperature and salinity profiles with a CTD at eight sites around the ASPSP. The depth of the surface mixed layer varied between 18-63 meters. Dinoflagellates dominated in terms of abundance representing over $80 \%$ and $65 \%$ of the individuals found in the surface and subsurface layers, respectively. A PERMANOVA test revealed significant differences $(p<0.001)$ among the dinoflagellate and tintinnid assemblages occurring in both layers, but no evidence for difference in the radiolarian assemblage was detected. Good ecological indicators were found mainly within the dinoflagellate assemblage. The thermocline induced stratification allowed the development of different microplanktonic protists assemblages above and under the depth of the surface mixed layer in the upper $100 \mathrm{~m}$ of the water column.
\end{abstract}

Key words: Dinoflagellates, ecological indicators, polycystine radiolarians, tintinnid ciliates, tropical oceans, vertical distribution.

\section{INTRODUCTION}

Tropical oceans are characterized by a strong and permanent thermocline, which inhibits the enrichment of the upper layers of the water column

Correspondence to: Alejandro Esteweson Santos Faustino da Costa

E-mail: alejandroesfc@gmail.com

* Contribution to the centenary of the Brazilian Academy of Sciences. by nutrient-rich deeper waters. Consequently, the upper layers of these ecosystems become depleted in nutrients in most of the extension of the tropical oceanic waters. Under such conditions, the "typical tropical structure" (Herbland and Voituriez 1979) is formed and maintained, being modified only with sufficient nutrient flux to the upper layers (Cullen 1982).

It is common knowledge that phytoplanktonic cells distribute unevenly throughout the water 
column in well stratified waters, flourishing in certain depths. Two striking features of oligotrophic oceans are the formation of a deep chlorophyll maximum (DCM) dominated by picophytoplanktonic cells and associated with the depth of the nitracline (Herbland et al. 1985, Partensky et al. 1996), and the occurrence of productivity maxima coinciding with or shallower than the depth of the DCM in regions with a permanent pycnocline (Longhurst and Harrison 1989). Diverse heterotrophic protists assemblages have been suggested as important consumers of the primary production, performing a very important role in the flow of energy within marine ecosystems, since they belong to a size class that is capable of feeding on pico- and nanoplanktonic cells and is still available to a wide range of metazoans (Sanders and Wickham 1993, Sherr and Sherr 1994, Calbet 2008) characterizing the so called microbial loop (Azam et al. 1983). These protists assemblages are commonly associated with the primary producers and thus their vertical distribution is not uniform as well.

The archipelago of São Pedro e São Paulo (ASPSP) is composed of serpentinized and mylonitized peridotites islets (Campos et al. 2010), originated from a major uplift of the lithospheric mantle (Maia et al. 2016). The archipelago is under the direct influence of two main current systems, the South Equatorial Current (SEC) flowing westward at the surface, and the Equatorial Under Current (EUC) flowing in the opposite direction at the subsurface. Albeit the commonness of enrichment processes in surface waters around oceanic islands - e.g. local upwelling and the development of Taylor columns, among others - no enrichment process has ever been observed around the ASPSP (Bröckel and Meyerhöfer 1999, Travassos et al. 1999). Consequently, the waters surrounding the archipelago are strongly stratified, with a surface mixed layer (SML) separated by a permanent thermocline - and associated pycnocline - from deeper nutrient-rich waters. However, the SML is considerably narrower around the ASPSP than in the surrounding oceanic waters. This raises the question if there is any change in the microplanktonic protists assemblages occurring in the narrow SML above the thermocline and in the subsurface layer (SSL) within the thermocline. We hypothesize that two assemblages may be found in the upper 100 meters depth of the water column, one typical of SML waters and other typical of SSL waters. If there is any change among these layers, indicator species of both layers shall be found. This is of great importance since studies related to the vertical distribution of microplanktonic protists assemblages in the tropical Atlantic are scarce (Dworetzky and Morley 1987, Boltovskoy et al. 1996).

\section{MATERIALS AND METHODS}

Samples were collected at seven depths $(1,10,20$, $30,50,75$, and $100 \mathrm{~m}$ ) of eight sites distributed at north ( $\mathrm{N}$ sites) and south (S sites) of the ASPSP (Figure 1a, b) during April and November 2015, the maximum and minimum rainfall period, respectively. A 10-L Niskin bottle was used to collect the samples. The collected volume was gently passed from the bottle to a PVC tube containing a $20 \mu \mathrm{m}$-mesh net fixed to its bottom to concentrate the sample on board of the "Transmar III" ship. The concentrates $(\sim 250 \mathrm{~mL})$ were fixated with lugol's iodine solution to a final concentration of 3\%. A SonTek CastAWAY CTD was used to profile temperature and salinity from the surface to a depth around 100 meters - the inferior limit of the equipment - in each site, to determine the depth of the top of the thermocline and to identify the water masses occurring at the sampled depths.

Prior to the analysis, the samples were left to settle, and their content was gently siphoned off. The whole volume of the concentrates was then analyzed using a Sedgwick-Rafter chamber and compound microscopy under a magnification of 100-200x to increase the representativeness of 
a

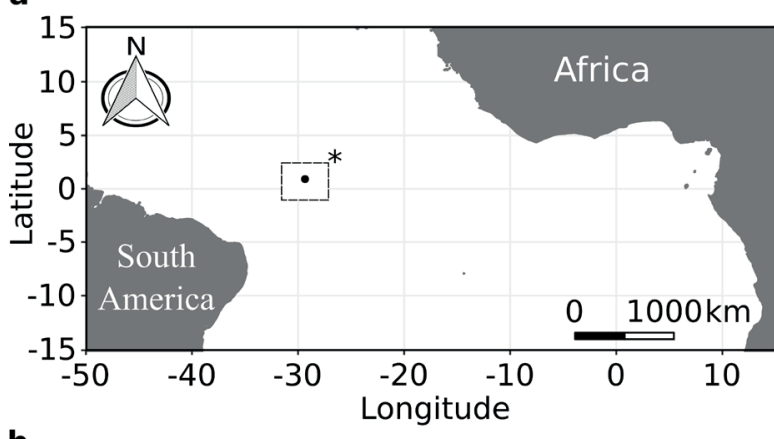

b

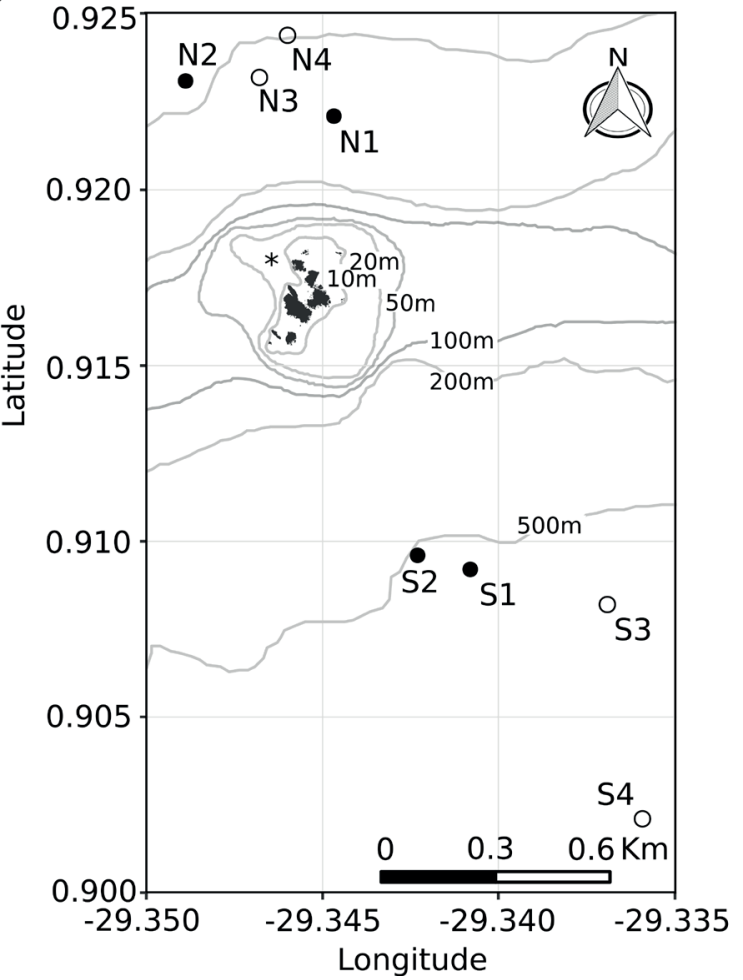

Figure 1 - Location of the ASPSP in the South Atlantic Ocean (a) and locations of the sampling sites around the archipelago (b). Filled circles represent the sites sampled in the period of maximum rainfall (April), and empty circles represent sites sampled during the period of minimum rainfall (November).

the sampling design. All the individuals in the samples were enumerated and identified to the lowest possible taxonomical unit according to classical taxonomic works - e.g. Wood (1963) and Balech (1988), for dinoflagellates; Haeckel (1887), and Boltovskoy (1999) for radiolarians; Kofoid and Campbell (1929) and Marshall (1969), for tintinnids. Foraminifers and aloricate ciliates were counted but not identified.
The dinoflagellates, tintinnid ciliates and polycystine radiolarian assemblages were investigated for differences among the SML and SSL by means of a PERMANOVA (Anderson 2001). The PERMANOVA was performed with basis on a Bray-Curtis distance matrix calculated after the transformation of the biological data by the $\ln (X+1)$. An unconstrained ordination of the samples based on the Bray-Curtis distance matrix calculated for each one of the considered assemblages was performed using the method of the principal coordinate analysis (PCoA). To investigate the existence of indicator species associated with the SML and SSL, an indicator value analysis (Dufrêne and Legendre 1997) was performed, and the significance of the relationships was tested by means of a permutation test.

We estimated the species richness and the diversity for the pooled data of each layer in each site. Species richness was calculated as the absolute number of species and as the diversity index of Margalef $\left(D_{M g}\right)$. The diversity was calculated as the dominance/uniformity index of Simpson $(D)$ and expressed as its reciprocal $1-D$, which is an effective diversity measure. The measurements of species richness and diversity were compared among the SML and SSL using the Mann-Whitney test.

All the statistical tests were performed using the R (R Core Team 2016) and RStudio Softwares.

\section{RESULTS}

\section{ENVIRONMENTAL DATA}

Sea surface temperature varied only between 27.2 ${ }^{\circ} \mathrm{C}$ at $\mathrm{N} 4$ and $\mathrm{S} 4$ during the period of minimal precipitation and $28.1{ }^{\circ} \mathrm{C}$ at $\mathrm{S} 2$ during the period of maximal precipitation. Sea surface salinity varied between 35.53 at $\mathrm{S} 3$ during the period of minimal precipitation, and 36.10 at $\mathrm{N} 2$ during the period of maximal precipitation. The depth of the onset of the thermocline varied between approx. $18 \mathrm{~m}$ at N1 during the period of maximal precipitation, and 63 
$\mathrm{m}$ at $\mathrm{S} 3$ during the period of minimal precipitation (see Table I). A maximum of salinity close to the depth of the beginning of the thermocline was observed in many of the sites. Two water masses were sampled during the study, the Tropical Surface Water (TSW) and the South Atlantic Central Water (SACW) (Figure 2).

\section{MICROPLANKTONIC PROTISTS ASSEMBLAGES}

The dinoflagellates were the most abundant (Figure 3 ) and diverse group (Table II) among the protists considered in this study; they represented $80.7 \%$ of the total number of individuals found in the SML, $65.8 \%$ of the individuals found in the SSL, and 124 identified species. The identification of very small and delicate forms, which did not preserve well, was hindered and thus this number is underestimated by some degree. Tintinnid and aloricate ciliates, as

TABLE I

Approximate depth of the onset of the thermocline at different sites and seasonal periods. Sites number 1 and 2 were sampled during the period of maximal precipitation,

while sites number 3 and 4 were sampled during the period of minimal precipitation.

\begin{tabular}{cccc}
\hline Site & Depth & Site & Depth \\
\hline N1 & $18 \mathrm{~m}$ & $\mathrm{~S} 1$ & $29 \mathrm{~m}$ \\
N2 & $30 \mathrm{~m}$ & $\mathrm{~S} 2$ & $34 \mathrm{~m}$ \\
N3 & $26 \mathrm{~m}$ & $\mathrm{~S} 3$ & $63 \mathrm{~m}$ \\
N4 & $30 \mathrm{~m}$ & $\mathrm{~S} 4$ & $59 \mathrm{~m}$ \\
\hline
\end{tabular}
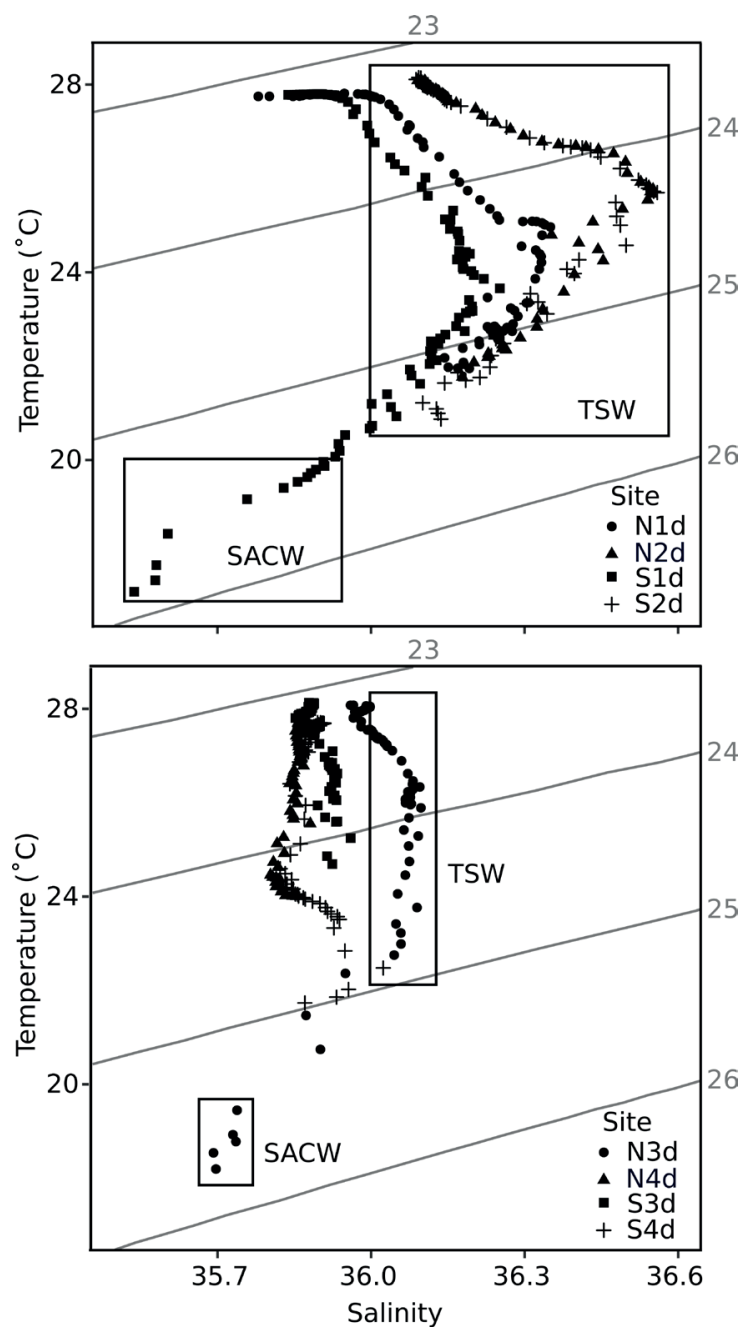

Figure 2 - T-S diagram of the stations performed during the period of maximal precipitation (N1, N2, S1 and S2) and minimal precipitation (N3, N4, S3 and S4). Different sites are displayed using different shapes according to the legend within the Figure.
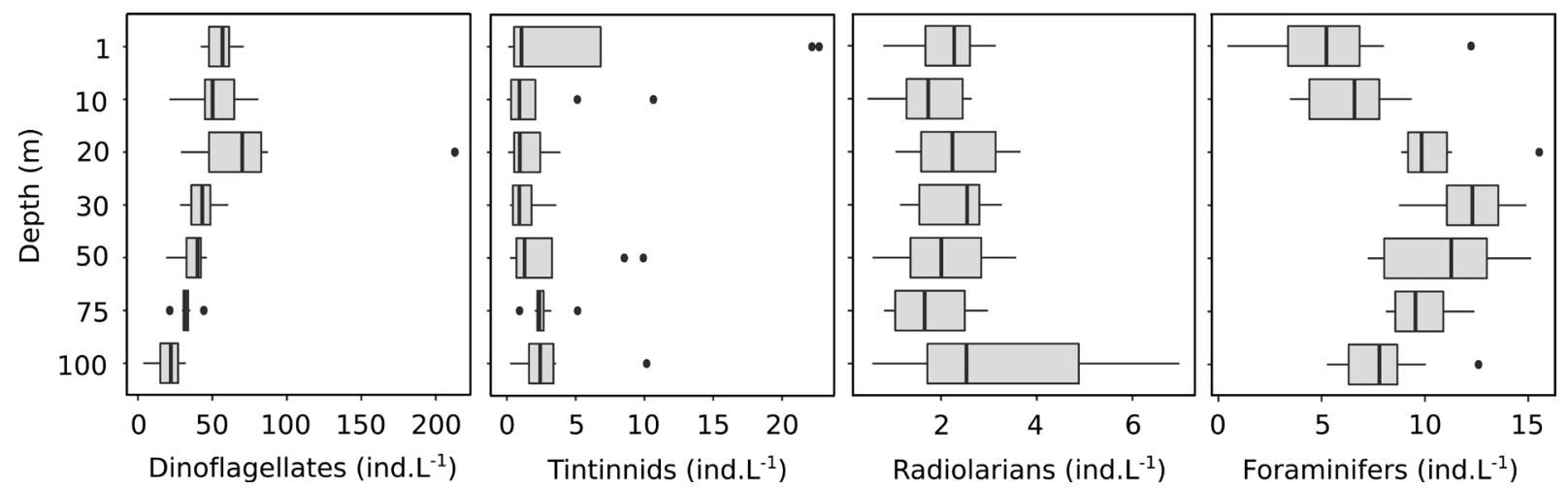

Figure 3 - Vertical distribution of the main microplanktonic protists assemblages in the upper 100 meters' depth of the water column around the ASPSP. 
TABLE II

List of identified species found in the surface mixed layer (SML) and subsurface layer (SSL) around the ASPSP. Species in bold were classified as ecological indicators by the IndVal analysis. The numbers are frequency of occurrence in the samples (\%).

\begin{tabular}{|c|c|c|c|c|c|}
\hline Species & SML & SSL & Species & SML & SSL \\
\hline \multicolumn{6}{|c|}{ Dinoflagellates } \\
\hline $\begin{array}{c}\text { Akashiwo sanguinea (Hirasaka) } \\
\text { Hansen \& Moestrup, } 2000\end{array}$ & 8.93 & 10.71 & $\begin{array}{l}\text { Phalacroma cuneus } \\
\text { Schütt, } 1895\end{array}$ & 1.79 & 16.07 \\
\hline $\begin{array}{l}\text { Amphisolenia bifurcata } \\
\text { Murray \& Whitting, } 1899\end{array}$ & 5.36 & 3.57 & $\begin{array}{c}\text { Phalacroma doryphorum } \\
\text { Stein, } 1883\end{array}$ & 10.71 & 14.29 \\
\hline $\begin{array}{l}\text { Amphisolenia bidentata } \\
\text { Schröder, } 1900\end{array}$ & 3.57 & 3.57 & $\begin{array}{c}\text { Phalacroma favus Kofoid \& } \\
\text { Michener, } 1911\end{array}$ & 5.36 & 7.14 \\
\hline $\begin{array}{l}\text { Amphisolenia globifera } \\
\text { Stein, } 1883\end{array}$ & - & 12.5 & $\begin{array}{c}\text { Phalacroma hindmarchii Murray \& } \\
\text { Whitting, } 1899\end{array}$ & - & 3.57 \\
\hline $\begin{array}{c}\text { Amphisolenia lemmermanni } \\
\text { Kofoid, } 1907\end{array}$ & 1.79 & - & $\begin{array}{l}\text { Phalacroma rapa } \\
\text { Jørgensen, } 1923\end{array}$ & 19.64 & 16.07 \\
\hline $\begin{array}{c}\text { Amphisolenia rectangulata } \\
\text { Kofoid, } 1907\end{array}$ & 3.57 & - & $\begin{array}{l}\text { Phalacroma rotundatum (Claparéde \& } \\
\text { Lachmann) Kofoid \& Michener, } 1911\end{array}$ & 48.21 & 51.79 \\
\hline $\begin{array}{l}\text { Amphisolenia schauinslandii } \\
\text { Lemmermann, } 1899\end{array}$ & 1.79 & - & Phalacroma sp. & - & 7.14 \\
\hline Amphisolenia sp. & 3.57 & 1.79 & Podolampas bipes Stein, 1883 & - & 1.79 \\
\hline $\begin{array}{l}\text { Balechina coerulea (Dogiel) } \\
\text { Taylor, } 1976\end{array}$ & 28.57 & 30.36 & Podolampas elegans Schütt, 1895 & 8.93 & 7.14 \\
\hline $\begin{array}{l}\text { Centrodinium pavillardii } \\
\text { Taylor, } 1976\end{array}$ & - & 1.79 & Podolampas palmipes Stein, 1883 & 48.21 & 50 \\
\hline $\begin{array}{c}\text { Ceratocorys armata (Schütt) } \\
\text { Kofoid, } 1910\end{array}$ & 7.14 & - & Podolampas spinifera Okamura, 1912 & 48.21 & 42.86 \\
\hline Ceratocorys horrida Stein, 1883 & 19.64 & 16.07 & $\begin{array}{l}\text { Prorocentrum balticum } \\
\text { (Lohmann) Loeblich, } 1970\end{array}$ & 48.21 & 50 \\
\hline $\begin{array}{l}\text { Ceratocorys reticulata } \\
\text { Graham, } 1942\end{array}$ & - & 1.79 & Prorocentrum gracile Schütt, 1895 & 42.86 & 41.07 \\
\hline Ceratocorys sp. & - & 3.57 & $\begin{array}{l}\text { Prorocentrum hoffmanianum } \\
\text { Faust, } 1990\end{array}$ & 1.79 & 3.57 \\
\hline Citharistes regius Stein, 1883 & 1.79 & - & $\begin{array}{c}\text { Prorocentrum micans } \\
\text { Ehrenberg, } 1834\end{array}$ & 41.07 & 46.43 \\
\hline $\begin{array}{c}\text { Cladopyxis brachiolata } \\
\text { Stein, } 1883\end{array}$ & 1.79 & 7.14 & $\begin{array}{l}\text { Prorocentrum rostratum } \\
\text { Stein, } 1883\end{array}$ & 3.57 & 1.79 \\
\hline $\begin{array}{c}\text { Margalefidinium flavum (Kofoid, 1931) } \\
\text { Gómez et al., } 2017\end{array}$ & 3.57 & - & Prorocentrum sp. & 3.57 & 5.36 \\
\hline $\begin{array}{c}\text { Corythodinium belgicae (Meunier) } \\
\text { Taylor, } 1976\end{array}$ & 3.57 & 5.36 & $\begin{array}{c}\text { Protoperidinium abei (Paulsen) } \\
\text { Balech, } 1974\end{array}$ & 5.36 & - \\
\hline $\begin{array}{c}\text { Corythodinium constrictum (Stein) } \\
\text { Taylor, } 1976\end{array}$ & 28.57 & 17.86 & $\begin{array}{l}\text { Protoperidinium cassum (Balech) } \\
\text { Balech, } 1974\end{array}$ & 48.21 & 44.64 \\
\hline $\begin{array}{c}\text { Corythodinium diploconus (Stein) } \\
\text { Taylor, } 1976\end{array}$ & - & 1.79 & $\begin{array}{l}\text { Protoperidinium curtipes } \\
\text { (Jørgensen) Balech, } 1974\end{array}$ & 46.43 & 30.36 \\
\hline
\end{tabular}


TABLE II (continuation)

\begin{tabular}{|c|c|c|c|c|c|}
\hline Species & SML & SSL & Species & SML & SSL \\
\hline $\begin{array}{c}\text { Corythodinium elegans (Pavillard) } \\
\text { Taylor, } 1976\end{array}$ & 17.86 & 10.71 & Protoperidinium sp. & 3.57 & 1.79 \\
\hline $\begin{array}{c}\text { Oxytoxum reticulatum (Stein) } \\
\text { Schütt, } 1899\end{array}$ & 21.43 & 28.57 & $\begin{array}{l}\text { Pyrocystis fusiformis } \\
\text { Thomson, } 1876\end{array}$ & 1.79 & 10.71 \\
\hline $\begin{array}{l}\text { Corythodinium tesselatum (Stein) } \\
\text { Loeblich \& Loeblich III, } 1966\end{array}$ & 46.43 & 19.64 & $\begin{array}{l}\text { Pyrocystis hamulus inaequalis } \\
\text { Schröder, } 1900\end{array}$ & 7.14 & 5.36 \\
\hline Dinophysis argus (Stein) Abé & 1.79 & - & $\begin{array}{c}\text { Pyrocystis pseudonoctiluca } \\
\text { Wyville-Thompson, } 1876\end{array}$ & 12.5 & 7.14 \\
\hline Dinophysis hastata Stein, 1883 & 12.5 & 8.93 & Pyrocystis robusta Kofoid, 1907 & 42.86 & 44.64 \\
\hline $\begin{array}{c}\text { Dinophysis ovata Claparéde \& } \\
\text { Lachmann, } 1859\end{array}$ & - & 1.79 & Pyrocystis sp. & 1.79 & 5.36 \\
\hline Dinophysis pusilla Jørgensen, 1923 & 26.79 & 21.43 & $\begin{array}{c}\text { Schuettiella mitra (Schütt) } \\
\text { Balech, } 1988\end{array}$ & 1.79 & - \\
\hline $\begin{array}{c}\text { Dinophysis schuetti Murray \& } \\
\text { Whitting, } 1899\end{array}$ & 10.71 & 30.36 & $\begin{array}{c}\text { Spiraulax kofoidii } \\
\text { Graham, } 1942\end{array}$ & 3.57 & 1.79 \\
\hline Dinophysis uracantha Stein, 1883 & 7.14 & 28.57 & $\begin{array}{c}\text { Tripos arietinus (Cléve) } \\
\text { Gómez, } 2013\end{array}$ & 3.57 & 1.79 \\
\hline Dinophysis sp. & 10.71 & 25 & $\begin{array}{l}\text { Tripos azoricus (Cleve) } \\
\text { Gómez, } 2013\end{array}$ & - & 1.79 \\
\hline Gonyaulax birostris Stein, 1883 & 33.93 & 26.79 & $\begin{array}{c}\text { Tripos candelabrus (Ehrenberg) } \\
\text { Gómez, } 2013\end{array}$ & 32.14 & 14.29 \\
\hline Gonyaulax pacifica Kofoid, 1907 & 23.21 & 17.86 & $\begin{array}{c}\text { Tripos concilians (Jørgensen) } \\
\text { Gómez, } 2013\end{array}$ & 3.57 & - \\
\hline Gonyaulax verior Sournia, 1973 & 12.5 & 1.79 & $\begin{array}{c}\text { Tripos contortus (Gourret) } \\
\text { Gómez, } 2013\end{array}$ & 3.57 & - \\
\hline Gonyaulax sp. & 48.21 & 51.79 & $\begin{array}{c}\text { Tripos declinatus (Karsten) } \\
\text { Gómez, } 2013\end{array}$ & 48.21 & 23.21 \\
\hline $\begin{array}{c}\text { Gymnodinium catenatum } \\
\text { Graham, } 1943\end{array}$ & 3.57 & 7.14 & $\begin{array}{l}\text { Tripos dens (Ostenfeld \& } \\
\text { Schmidt) Gómez, } 2013\end{array}$ & 1.79 & - \\
\hline Gymnodinium sp. & 37.5 & 16.07 & $\begin{array}{l}\text { Tripos digitatus (Schütt) } \\
\text { Gómez, } 2013\end{array}$ & - & 1.79 \\
\hline Heterodinium sp. & - & 3.57 & $\begin{array}{c}\text { Tripos euarcuatus (Jørgensen) } \\
\text { Gómez, } 2013\end{array}$ & 3.57 & 1.79 \\
\hline $\begin{array}{l}\text { Histioneis crateriformis } \\
\text { Stein, } 1883\end{array}$ & 8.93 & 5.36 & $\begin{array}{c}\text { Tripos falcatiformis (Jørgensen) } \\
\text { Gómez, } 2013\end{array}$ & 1.79 & 1.79 \\
\hline $\begin{array}{c}\text { Histioneis cymbalaria } \\
\text { Stein, } 1883\end{array}$ & 3.57 & 10.71 & $\begin{array}{c}\text { Tripos fusus (Ehrenberg) } \\
\text { Gómez, } 2013\end{array}$ & 37.5 & 21.43 \\
\hline $\begin{array}{c}\text { Histioneis milneri Murray \& } \\
\text { Whitting, } 1899\end{array}$ & 48.21 & 10.71 & $\begin{array}{c}\text { Tripos geniculatus (Lemmermann) } \\
\text { Gómez, } 2013\end{array}$ & - & 3.57 \\
\hline $\begin{array}{c}\text { Histioneis hyalina Kofoid \& } \\
\text { Michener, } 1911\end{array}$ & 26.79 & 26.79 & $\begin{array}{c}\text { Tripos gibberus (Gourret) } \\
\text { Gómez, } 2013\end{array}$ & 23.21 & 12.5 \\
\hline Histioneis isselii Forti, 1932 & 3.57 & - & $\begin{array}{c}\text { Tripos horridus (Cleve) } \\
\text { Gómez, } 2013\end{array}$ & 10.71 & 12.5 \\
\hline
\end{tabular}


TABLE II (continuation)

\begin{tabular}{|c|c|c|c|c|c|}
\hline Species & SML & SSL & Species & SML & SSL \\
\hline $\begin{array}{c}\text { Histioneis panaria Kofoid \& } \\
\text { Skogsberg, } 1928\end{array}$ & - & 1.79 & $\begin{array}{c}\text { Tripos kofoidii (Jørgensen) } \\
\text { Gómez, } 2013\end{array}$ & 30.36 & 32.14 \\
\hline $\begin{array}{c}\text { Histioneis striata Kofoid \& } \\
\text { Michener, } 1911\end{array}$ & 3.57 & 10.71 & $\begin{array}{c}\text { Tripos lineatus (Ehrenberg) } \\
\text { Gómez, } 2013\end{array}$ & 7.14 & 1.79 \\
\hline Histioneis sp. & 7.14 & 7.14 & $\begin{array}{c}\text { Tripos limulus (Pouchet) } \\
\text { Gómez, } 2013\end{array}$ & 1.79 & 1.79 \\
\hline Noctiluca sp. & 17.86 & 1.79 & $\begin{array}{c}\text { Tripos longissimus (Schröder) } \\
\text { Gómez, } 2013\end{array}$ & - & 1.79 \\
\hline $\begin{array}{c}\text { Ornithocercus cristatus } \\
\text { Matzenauer, } 1933\end{array}$ & 5.36 & 5.36 & $\begin{array}{c}\text { Tripos lunula (Schimper ex } \\
\text { Karsten) Gómez, } 2013\end{array}$ & 1.79 & 1.79 \\
\hline $\begin{array}{c}\text { Ornithocercus magnificus } \\
\text { Stein, } 1883\end{array}$ & 30.36 & 28.57 & $\begin{array}{c}\text { Tripos macroceros (Ehrenberg) } \\
\text { Gómez, } 2013\end{array}$ & 21.43 & 8.93 \\
\hline $\begin{array}{c}\text { Ornithocercus quadratus } \\
\text { Schütt, } 1900\end{array}$ & 25 & 17.86 & $\begin{array}{c}\text { Tripos massiliensis (Gourret) } \\
\text { Gómez, } 2013\end{array}$ & 1.79 & 1.79 \\
\hline Ornithocercus steinii Schütt, 1900 & 17.86 & 7.14 & $\begin{array}{c}\text { Tripos muelleri Bory de } \\
\text { Saint-Vicent, } 1824\end{array}$ & 21.43 & 3.57 \\
\hline $\begin{array}{l}\text { Ornithocercus thumi (Schmidt) } \\
\text { Kofoid \& Skogsberg, } 1928\end{array}$ & 3.57 & - & $\begin{array}{c}\text { Tripos pentagonus (Gourret) } \\
\text { Gómez, } 2013\end{array}$ & 48.21 & 28.57 \\
\hline Ornithocercus sp. & 10.71 & 12.5 & $\begin{array}{c}\text { Tripos platycornis (Daday) } \\
\text { Gómez, } 2013\end{array}$ & - & 1.79 \\
\hline $\begin{array}{l}\text { Oxytoxum elongatum } \\
\text { Wood, } 1963\end{array}$ & 21.43 & 19.64 & $\begin{array}{c}\text { Tripos praelongus (Lemmermann) } \\
\text { Gómez, } 2013\end{array}$ & - & 1.79 \\
\hline Oxytoxum laticeps Schiller, 1937 & - & 1.79 & $\begin{array}{c}\text { Tripos pulchellus (Schröder) } \\
\text { Gómez, } 2013\end{array}$ & 1.79 & - \\
\hline $\begin{array}{c}\text { Oxytoxum milneri Murray \& } \\
\text { Whitting, } 1899\end{array}$ & 25 & 23.21 & $\begin{array}{c}\text { Tripos ranipes (Cleve) } \\
\text { Gómez, } 2013\end{array}$ & 1.79 & 5.36 \\
\hline Oxytoxum ovum Gaarder, 1954 & 1.79 & 1.79 & $\begin{array}{l}\text { Tripos tenuis (Ostenfeld \& } \\
\text { Schmidt) Gómez, } 2013\end{array}$ & 1.79 & - \\
\hline $\begin{array}{c}\text { Oxytoxum robustum Kofoid \& } \\
\text { Michener, } 1911\end{array}$ & - & 1.79 & $\begin{array}{c}\text { Tripos teres (Kofoid) } \\
\text { Gómez, } 2013\end{array}$ & 48.21 & 25 \\
\hline $\begin{array}{c}\text { Oxytoxum sceptrum (Stein) } \\
\text { Schröder, } 1906\end{array}$ & 17.86 & 10.71 & $\begin{array}{l}\text { Tripos vultur (Cleve) } \\
\text { Gómez, } 2013\end{array}$ & 10.71 & 5.36 \\
\hline Oxytoxum scolopax Stein, 1883 & 46.43 & 50 & Tripos sp. & 28.57 & 17.86 \\
\hline Oxytoxum turbo Kofoid, 1907 & - & 5.36 & $\begin{array}{c}\text { Triposolenia depressa } \\
\text { Kofoid, } 1906\end{array}$ & - & 17.86 \\
\hline $\begin{array}{l}\text { Oxytoxum sphaeroideum } \\
\text { Stein, } 1883\end{array}$ & 3.57 & - & $\begin{array}{c}\text { Tryblionella compressa (Bailey) } \\
\text { Poulin, } 1990\end{array}$ & 46.43 & 46.43 \\
\hline Oxytoxum $\mathrm{sp}$. & - & 3.57 & Warnowia sp. & 5.36 & 12.5 \\
\hline \multicolumn{6}{|c|}{ Tintinnids } \\
\hline $\begin{array}{c}\text { Codonaria cistellula (Fol) Kofoid } \\
\text { \& Campbell, } 1929\end{array}$ & 1.79 & 1.79 & $\begin{array}{c}\text { Proplectella praelonga Kofoid } \\
\text { \& Campbell, } 1929\end{array}$ & 8.93 & 5.36 \\
\hline $\begin{array}{c}\text { Codonella acuta Kofoid } \\
\text { \& Campbell, } 1929\end{array}$ & 12.5 & 8.93 & $\begin{array}{c}\text { Protorhabdonella simplex (Cleve) } \\
\text { Jörgensen, } 1924\end{array}$ & - & 1.79 \\
\hline
\end{tabular}


TABLE II (continuation)

\begin{tabular}{|c|c|c|c|c|c|}
\hline Species & SML & SSL & Species & SML & SSL \\
\hline $\begin{array}{l}\text { Codonella amphorella } \\
\text { Biedermann, } 1893\end{array}$ & - & 8.93 & $\begin{array}{c}\text { Rhabdonellopsis apophysata (Cleve) } \\
\text { Kofoid \& Campbell, } 1929\end{array}$ & - & 1.79 \\
\hline $\begin{array}{c}\text { Codonella apicata Kofoid \& } \\
\text { Campbell, } 1929\end{array}$ & 17.86 & 16.07 & $\begin{array}{l}\text { Steenstrupiella steenstrupii (Claparède \& } \\
\text { Lachmann) Kofoid \& Campbell, } 1929\end{array}$ & 1.79 & - \\
\hline Codonella galea Haeckel, 1873 & 14.29 & 30.36 & $\begin{array}{l}\text { Stenosemella avellana (Meunier) } \\
\text { Kofoid \& Campbell, } 1929\end{array}$ & 5.36 & 1.79 \\
\hline Codonella relicta Minkiewich, 1905 & 3.57 & 3.57 & $\begin{array}{c}\text { Stenosemella inflata Kofoid } \\
\text { \& Campbell, } 1929\end{array}$ & 1.79 & - \\
\hline Codonella sp. & 5.36 & 1.79 & Stenosemella nivalis Meunier, 1910 & - & 5.36 \\
\hline $\begin{array}{c}\text { Codonellopsis orthoceras (Haeckel) } \\
\text { Jörgensen, } 1924\end{array}$ & 3.57 & 12.5 & Stenosemella steinii Jörgensen, 1912 & 1.79 & 1.79 \\
\hline $\begin{array}{c}\text { Climacocylis scalaroides Kofoid \& } \\
\text { Campbell, } 1929\end{array}$ & 1.79 & - & $\begin{array}{l}\text { Stenosemella ventricosa (Claparède } \\
\text { \& Lachmann) Jörgensen, } 1924\end{array}$ & 7.14 & 5.36 \\
\hline $\begin{array}{l}\text { Dadayiella ganymedes (Entz) } \\
\text { Kofoid \& Campbell, } 1929\end{array}$ & 1.79 & 1.79 & Stenosemella sp. & 5.36 & 12.5 \\
\hline $\begin{array}{c}\text { Dictyocysta californiensis Kofoid } \\
\text { \& Campbell, } 1929\end{array}$ & - & 3.57 & Tintinnopsis acuminata Daday, 1887 & 1.79 & 3.57 \\
\hline Dictyocysta duplex Brandt, 1906 & 1.79 & 3.57 & Tintinnopsis beroidea Stein, 1867 & 7.14 & 8.93 \\
\hline Dictyocysta lepida Ehrenberg, 1854 & 16.07 & 30.36 & $\begin{array}{c}\text { Tintinnopsis brasiliensis Kofoid } \\
\text { \& Campbell, } 1929\end{array}$ & 14.29 & 16.07 \\
\hline Dictyocysta entzi Jörgensen, 1924 & - & 14.29 & $\begin{array}{l}\text { Tintinnopsis rotundata Kofoid } \\
\quad \& \text { Campbell, } 1929\end{array}$ & 16.07 & 17.86 \\
\hline $\begin{array}{l}\text { Dictyocysta extensa Kofoid } \\
\text { \& Campbell, } 1929\end{array}$ & 1.79 & 10.71 & Tintinnopsis minuta Wailes, 1925 & - & 1.79 \\
\hline Dictyocysta mitra Haeckel, 1873 & 3.57 & 23.21 & Tintinnopsis nana Lohmann, 1908 & 3.57 & 3.57 \\
\hline Dictyocysta sp. & 8.93 & 26.79 & Tintinnopsis parva Merkle, 1909 & 1.79 & - \\
\hline $\begin{array}{l}\text { Epiplocylis blanda (Jörgensen) } \\
\text { Kofoid \& Campbell, } 1939\end{array}$ & 1.79 & - & Tintinnopsis sp. & 7.14 & 5.36 \\
\hline $\begin{array}{l}\text { Eutintinnus fraknoi (Daday) } \\
\text { Kofoid \& Campbell, } 1939\end{array}$ & 1.79 & 3.57 & Undella claparedei (Entz) Daday, 1887 & 1.79 & 1.79 \\
\hline $\begin{array}{l}\text { Eutintinnus lusus-undae (Entz) } \\
\text { Kofoid \& Campbell, } 1939\end{array}$ & 1.79 & - & Undella hyalina Daday, 1887 & - & 1.79 \\
\hline $\begin{array}{l}\text { Eutintinnus tubulosus (Ostenfeld) } \\
\text { Kofoid \& Campbell, } 1939\end{array}$ & 8.93 & 17.86 & Undella subacuta Cleve, 1900 & 1.79 & - \\
\hline Eutintinnus sp. & 7.14 & 1.79 & Undella sp. & 1.79 & 3.57 \\
\hline $\begin{array}{l}\text { Poroecus curtus Kofoid } \\
\text { \& Campbell, } 1929\end{array}$ & - & 5.36 & & & \\
\hline \multicolumn{6}{|c|}{ Radiolarians } \\
\hline Acanthosphaera actinota Haeckel, 1860 & 1.79 & 7.14 & Hexacontium sp. & - & 1.79 \\
\hline Acrobotrys sp. & - & 1.79 & Hexapyle sp. & 3.57 & 5.36 \\
\hline $\begin{array}{c}\text { Actinomma leptodermum (Jörgensen) } \\
\text { Nigrini \& Moore, } 1979\end{array}$ & - & 1.79 & Hexastylus phaenaxonius & 1.79 & 1.79 \\
\hline Actinomma sp. & 10.71 & 7.14 & Hymeniastrum sp. & 1.79 & 3.57 \\
\hline
\end{tabular}


TABLE II (continuation)

\begin{tabular}{|c|c|c|c|c|c|}
\hline Species & SML & SSL & Species & SML & SSL \\
\hline Amphiplecta sp. & 8.93 & 14.29 & Lamprocyclas sp. & 8.93 & 7.14 \\
\hline Amphispyris toxarium & - & 1.79 & Lampromitra sp. & 5.36 & 3.57 \\
\hline Amphispyris sp. & 1.79 & 3.57 & Larcopyle sp. & 1.79 & 1.79 \\
\hline Amphitholus sp. & 1.79 & 1.79 & $\begin{array}{c}\text { Larcospira quadrangular } \\
\text { Haeckel, } 1887\end{array}$ & - & 3.57 \\
\hline Antarctissa sp. & - & 1.79 & $\begin{array}{c}\text { Lithomelissa thoracites } \\
\text { Haeckel, } 1860\end{array}$ & 1.79 & 5.36 \\
\hline Arachnocorys sp. & 1.79 & 3.57 & Lithomelissa sp. & 28.57 & 42.86 \\
\hline Botryopyle dictyocephalus Haeckel, 1887 & - & 1.79 & $\begin{array}{c}\text { Lophophaena buetschlii (Haeckel) } \\
\text { Petrushevskaya, } 1971\end{array}$ & - & 1.79 \\
\hline Carpocanistrum sp. & 1.79 & 1.79 & $\begin{array}{c}\text { Lophophaena hispida (Ehrenberg) } \\
\text { Petrushevskaya, } 1971\end{array}$ & 10.71 & 17.86 \\
\hline Carpocanium sp. & - & 1.79 & Octopyle sp. & - & 1.79 \\
\hline Ceratospyris sp. & 1.79 & - & Peromelissa sp. & 1.79 & 1.79 \\
\hline $\begin{array}{c}\text { Cladoscenium tricolpium (Haeckel) } \\
\text { Jörgensen, } 1900\end{array}$ & - & 1.79 & Plegmosphaera sp. & - & 1.79 \\
\hline $\begin{array}{c}\text { Corythospyris stapedius (Haeckel) } \\
\text { Goll, } 1978\end{array}$ & 1.79 & - & Pseudocubus obeliscus Haeckel, 1887 & 1.79 & 1.79 \\
\hline Clathrocorys sp. & - & 1.79 & Pterocanium charybdeum (Müller) & - & 1.79 \\
\hline Clathrocyclas sp. & 1.79 & 1.79 & $\begin{array}{c}\text { Pterocorys minythorax (Nigrini) } \\
\text { Nigrini \& Moore, } 1979\end{array}$ & 7.14 & 8.93 \\
\hline $\begin{array}{l}\text { Stichopilidium kruegeri (Popofsky) } \\
\text { Nishimura \& Yamauchi, } 1984\end{array}$ & - & 1.79 & $\begin{array}{c}\text { Pterocorys zancleus (Müller) } \\
\text { Nigrini \& Moore, } 1979\end{array}$ & 3.57 & - \\
\hline Cycladophora sp. & 26.79 & 21.43 & Pteroscenium pinnatum Haeckel, 1887 & - & 3.57 \\
\hline Cyrtolagena laguncula Haeckel, 1887 & - & 1.79 & Saccospyris sp. & - & 1.79 \\
\hline Dictyocephalus sp. & - & 1.79 & Spirocyrtis scalaris Haeckel, 1887 & 1.79 & 5.36 \\
\hline $\begin{array}{c}\text { Dictyocoryne truncatum (Ehrenberg) } \\
\text { Nigrini \& Moore, } 1979\end{array}$ & 5.36 & 3.57 & Spongaster tetras Ehrenberg, 1860 & 5.36 & 5.36 \\
\hline Dictyocoryne sp. & - & 1.79 & Spongotrochus sp. & 8.93 & 3.57 \\
\hline $\begin{array}{c}\text { Pseudodictyophimus gracilipes } \\
\text { Caulet, } 1979\end{array}$ & - & 3.57 & Tetrapyle octacantha Müller, 1858 & 19.64 & 23.21 \\
\hline Dictyophimus infabricatus Nigrini, 1968 & - & 3.57 & $\begin{array}{c}\text { Thecosphaera inermis (Haeckel) } \\
\text { Haeckel, } 1887\end{array}$ & 1.79 & 1.79 \\
\hline Dictyophimus sp. & 3.57 & 3.57 & Theopilium tricostatum Haeckel, 1887 & 1.79 & - \\
\hline Discopyle sp. & 5.36 & 8.93 & Tholospira cervicornis Haeckel, 1887 & 3.57 & 1.79 \\
\hline $\begin{array}{c}\text { Euchitonia elegans-furcata (Ehrenberg) } \\
\text { Boltovskoy, } 1998\end{array}$ & - & 1.79 & Tholospyris sp. & 12.5 & 7.14 \\
\hline Euchitonia sp. & - & 1.79 & Zygocampe chrysalidium Haeckel, 1887 & 12.5 & 5.36 \\
\hline Eucoronis sp. & 3.57 & - & Zygocircus productus Bütschli, 1882 & 8.93 & 5.36 \\
\hline $\begin{array}{c}\text { Eucyrtidium acuminatum (Ehrenberg) } \\
\text { Ehrenberg, } 1847\end{array}$ & 1.79 & 8.93 & Zygocircus sp. & - & 1.79 \\
\hline Eucyrtidium sp. & 1.79 & 1.79 & & & \\
\hline
\end{tabular}


well as radiolarians and foraminifers were present in relatively low numbers in comparison with dinoflagellates. The tintinnid ciliates represented $3.9 \%$ of the individuals found in the SML, $6 \%$ of the individuals found in the SSL, and a total of 45 identified species. Radiolarians represented 2.8\% of the individuals found in the SML, $5 \%$ of the individuals counted in the SSL, and a total of 65 identified species. Foraminifers represented $10.4 \%$ of the individuals in the SML, and $21.5 \%$ of the individuals found in the SSL.

\section{SURFACE MIXED LAYER (SML), SUBSURFACE LAYER (SSL), AND INDICATOR SPECIES}

The difference among the SML and SSL was very conspicuous in the dinoflagellate assemblage (PERMANOVA, $p<0.001$; See Table III and Figure $4 a)$ and was also observed in the tintinnid ciliates assemblage (PERMANOVA, $p<0.001$; See Table III and Figure $4 b$ ). In both cases, the difference among the two layers was independent of the rainfall regime being considered. No evidence for

TABLE III

Results of the PERMANOVA analyses performed on the Bray-Curtis distance matrices of the dinoflagellate, tintinnid ciliates, and radiolarian assemblages in the SML and SSL layers.

\begin{tabular}{cccccc}
\hline Source & d.f. & $\boldsymbol{S S}$ & $\boldsymbol{M S}$ & $\boldsymbol{F}$ & $\boldsymbol{p}$ \\
\hline \multicolumn{5}{c}{ Dinoflagellates } \\
\hline Layer & 1 & 1.73 & 1.73 & 11.20 & $\mathbf{0 . 0 0 1}$ \\
Residuals & 54 & 8.34 & 0.16 & & \\
Total & 55 & 10.07 & & \\
\hline \multicolumn{6}{c}{ Tintinnids } \\
\hline Layer & 1 & 2.22 & 2.22 & 2.96 & $\mathbf{0 . 0 0 0 4}$ \\
Residuals & 54 & 40.51 & 0.75 & & \\
Total & 55 & 42.74 & & \\
\hline \multicolumn{6}{c}{ Radiolarians } \\
Layer & 1 & 0.74 & 0.74 & 1.55 & 0.06 \\
Residuals & 54 & 25.74 & 0.48 \\
Total & 55 & 26.47 & & \\
\hline
\end{tabular}

Source - sources of variation; d.f. - degrees of freedom; SS sequential sums of squares; MS - mean squares; $\mathrm{F}$ - $F$ statistics; $\mathrm{p}-p$ value.

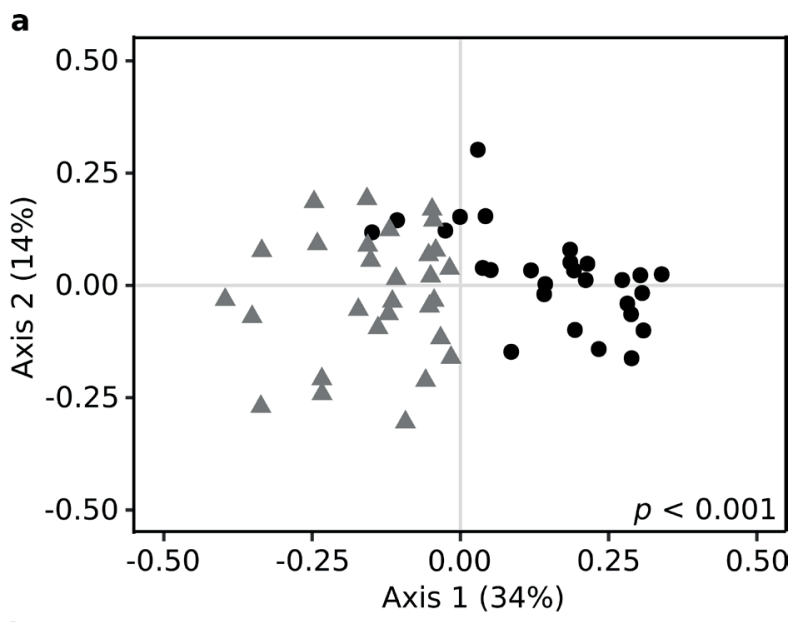

b

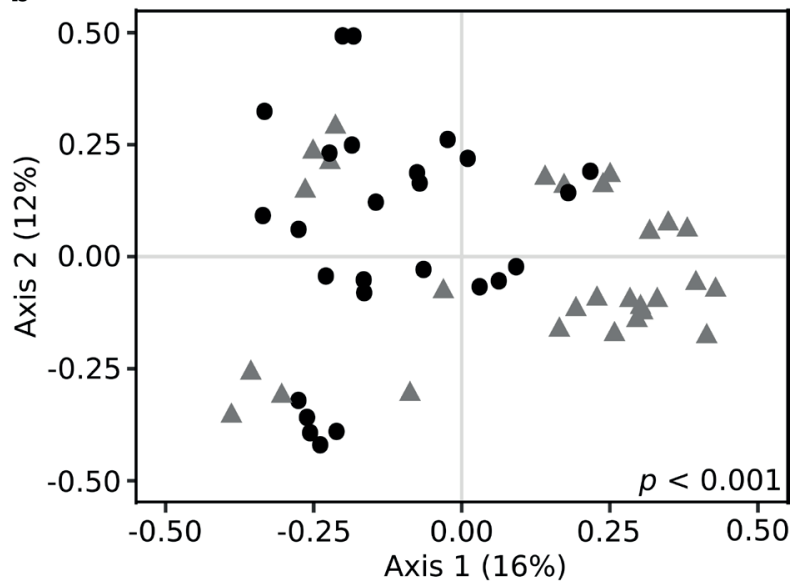

C

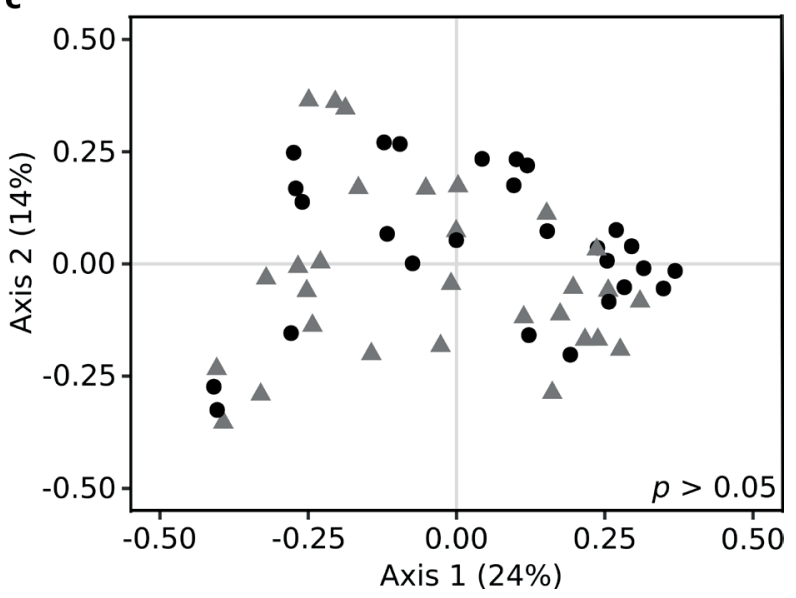

Figure 4 - PCoA unconstrained ordination using the biological data of the dinoflagellate assemblage (a), tintinnid ciliates assemblage (b), and polycystine radiolarians assemblage (c) The black circles represent samples collected in the surface mixed layer (SML) and the gray triangles represent samples collected in the subsurface layer (SSL). The $p$ value of a PERMANOVA comparing each assemblage in both layers is also displayed. 
differences in the radiolarian assemblage among the two layers was found (Table III and Figure 4c) during any of the rainfall regimes. The PCoA ordination of the dinoflagellate assemblage exhibited the clearest distinction among the SML and SSL waters. A total of 20 indicator species of dinoflagellates were found; 15 for the SML and five for the SSL waters (Table IV). Five indicator species of tintinnid ciliates were found for the SSL waters (Table IV), but no tintinnid indicator of the SML was found. No radiolarian species was found to be indicator of the SML or SSL waters within the depth interval sampled. Vertical profiles of two SML and two SSL indicator species are presented in Figure 5.

\section{SPECIES RICHNESS AND DIVERSITY}

The influence of the thermocline in the species richness and diversity differed among the considered protists assemblages. In the dinoflagellate assemblage, no difference in the species richness $\left(D_{M G}\right)$ was found among both layers. Nevertheless, the absolute number of species found in each layer was significantly different with higher mean values in the SML (t test, $p<0.01$ ). Species diversity, as the Simpson index $(D)$ was also different among both layers, with a higher mean value in the SML ( $t$ test, $p<0.001)$. In the tintinnid ciliates assemblage only the absolute number of species was different among the layers, with a higher mean value in the $\mathrm{SSL}$ ( $\mathrm{t}$ test, $p<0.01$ ). No difference in the absolute number of species, species richness $\left(D_{M G}\right)$, and diversity $(D)$ among both layers was detected in the radiolarian assemblage. Table $\mathrm{V}$ displays mean values along with standard deviation for the absolute number of species found in each layer, species richness, and diversity of the three protists assemblages considered.

\section{DISCUSSION}

\section{HYDROLOGY}

The ASPSP is located at very low latitudes, being characterized by the presence of a permanent

TABLE IV

Indicator species determined by the Indicator Value analysis.

\begin{tabular}{cccccccccc}
\hline \multicolumn{1}{c}{ Surface Mixed Layer (SML) } & \multicolumn{7}{c}{ Subsurface Layer (SSL) } \\
\hline Species & A & B & stat & $\boldsymbol{p}$ & Species & A & B & stat & $\boldsymbol{p}$ \\
\hline Tripos declinatus & 0.94 & 1 & 0.97 & 0.001 & Dictyocysta lepida & 0.93 & 0.59 & 0.74 & 0.004 \\
Tripos teres & 0.93 & 1 & 0.96 & 0.001 & Dictyocysta sp. & 0.93 & 0.52 & 0.70 & 0.002 \\
Tripos pentagonus & 0.90 & 1 & 0.95 & 0.001 & Dinophysis uracantha & 0.86 & 0.55 & 0.69 & 0.001 \\
Histioneis milneri & 0.88 & 1 & 0.94 & 0.001 & Dinophysis schuetti & 0.78 & 0.59 & 0.67 & 0.003 \\
Corythodinium tesselatum & 0.85 & 0.96 & 0.90 & 0.001 & Dictyocysta mitra & 0.97 & 0.45 & 0.66 & 0.002 \\
Protoperidinium curtipes & 0.83 & 0.96 & 0.90 & 0.001 & Triposolenia depressa & 1 & 0.35 & 0.59 & 0.001 \\
Tripos fusus & 0.81 & 0.78 & 0.79 & 0.001 & Phalacroma cuneus & 0.91 & 0.31 & 0.53 & 0.008 \\
Tripos candelabrus & 0.83 & 0.67 & 0.75 & 0.001 & Dictyocysta entzi & 1 & 0.28 & 0.53 & 0.004 \\
Gymnodinium sp. & 0.70 & 0.78 & 0.74 & 0.002 & Tintinnina n.i. & 0.83 & 0.31 & 0.51 & 0.021 \\
Tripos muelleri & 0.93 & 0.44 & 0.64 & 0.001 & Amphisolenia globifera & 1 & 0.24 & 0.49 & 0.010 \\
Tripos macroceros & 0.89 & 0.44 & 0.63 & 0.006 & Codonella amphorella & 1 & 0.17 & 0.41 & 0.045 \\
Tripos gibberus & 0.80 & 0.48 & 0.62 & 0.014 & & & &
\end{tabular}

A - specificity component of the species to the sample group; B - fidelity or sensitivity component of the species to the sample group; Stat - Indicator Value for the species; $p$ - uncorrected permutation probability; Tintinnina n.i. - non-identified tintinnid ciliate. 

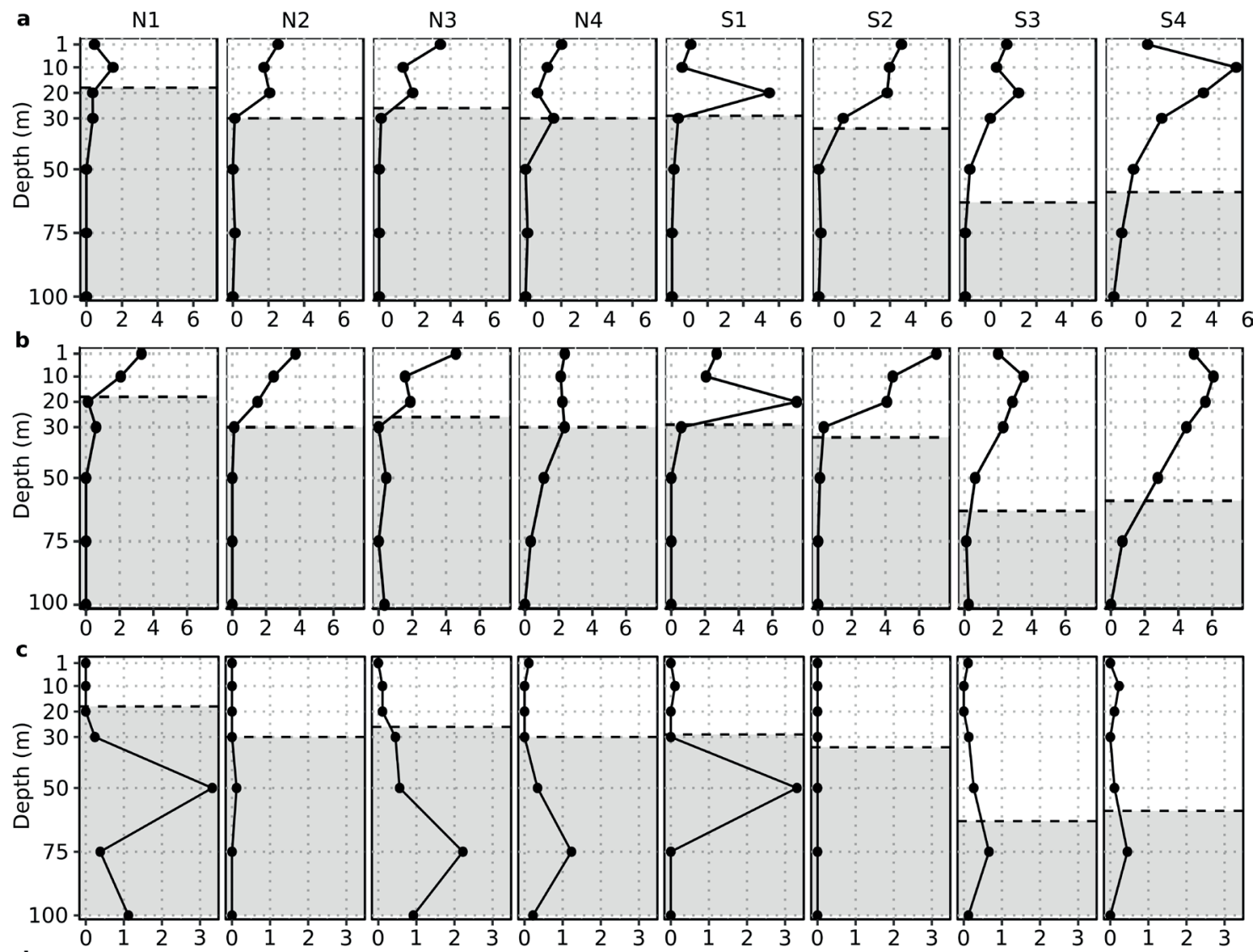

d

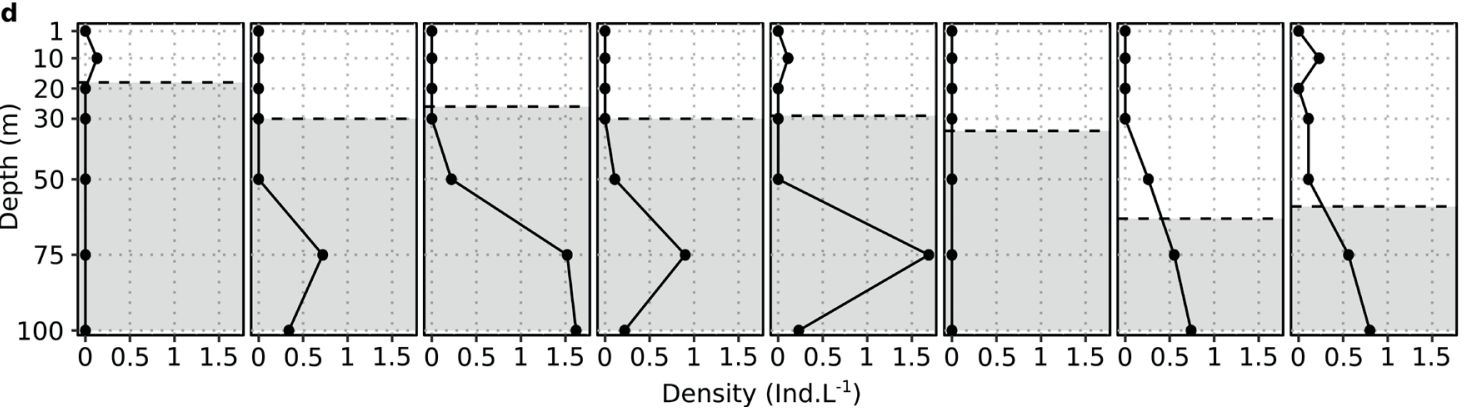

Figure 5 - Vertical profile of two indicator species of the SML, the dinoflagellate Tripos declinatus (a) and the dinoflagellate Tripos teres (b); and two indicator species of the SSL, the tintinnid ciliate Dictyocysta lepida (c) and a non-identified Dictyocysta tintinnid ciliate (d). The SML was indicated exclusively by dinoflagellates, and the SSL by dinoflagellates and tintinnid ciliates.

TABLE V

Absolute number of species (n), Margalef species richness $\left(D_{M G}\right)$, and Simpson diversity index $(D)$ for the assemblages of the dinoflagellates, tintinnid ciliates and polycystine radiolarians. Mean values \pm standard deviation.

\begin{tabular}{ccccccc}
\hline & \multicolumn{3}{c}{ SML } & \multicolumn{3}{c}{ SSL } \\
\cline { 2 - 7 } & $\mathrm{n}$ & $D_{M G}$ & $D$ & $\mathrm{n}$ & $D_{M G}$ & $D$ \\
\hline Dinoflagellates & $\mathbf{3 6} \pm \mathbf{7}^{* *}$ & $5.91 \pm 1.1$ & $\mathbf{0 . 9 2} \pm \mathbf{0 . 0 2} * * *$ & $\mathbf{2 9} \pm \mathbf{1 0} * *$ & $5.49 \pm 1.4$ & $\mathbf{0 . 8 5} \pm \mathbf{0 . 0 8 * * *}$ \\
Tintinnids & $\mathbf{4} \pm \mathbf{3}^{* *}$ & $1.54 \pm 0.64$ & $0.59 \pm 0.26$ & $\mathbf{7} \pm \mathbf{3}^{* *}$ & $1.83 \pm 0.84$ & $0.64 \pm 0.25$ \\
Radiolarians & $6 \pm 2$ & $2.13 \pm 0.68$ & $0.75 \pm 0.1$ & $7 \pm 3$ & $2.42 \pm 0.73$ & $0.75 \pm 0.1$ \\
\hline
\end{tabular}

Significant at the level of 0.05 ; * significant at the level of $0.01 ; * *$ significant at the level of 0.001 . 
thermocline, which inhibits the enrichment of superficial waters by nutrient rich deeper waters. Some authors have already investigated possible mechanisms of bottom-induced upwelling around the archipelago leading to the enrichment of the superficial waters and enhancement of the phytoplanktonic production, but such a process was never found in the area (Bröckel and Meyerhöfer 1999, Travassos et al. 1999).

Based on T-S pairs, at least two water masses were sampled during the study, the TSW and the SACW. The TSW forms the mixed layer of the tropical Atlantic Ocean and its lower limit is represented by the $20{ }^{\circ} \mathrm{C}$ isotherm (Stramma and Schott 1999). According to the criterion of Stramma and Schott (1999), the TSW may be identified as water with temperature higher than $20{ }^{\circ} \mathrm{C}$ and salinity of at least 36 . In both sampling periods - maximal and minimal precipitation water of temperature above $20^{\circ} \mathrm{C}$ and salinity under 36 were observed in the upper layer of the water column. This water is out of the typical salinity of the TSW according to Stramma and Schott (1999) and was already observed in the archipelago area (Travassos et al. 1999, Medeiros et al. 2009a, b). High levels of precipitation are associated with the location of the ASPSP on very low latitudes at the Intertropical Convergence Zone, which contributes to the slight dilution of the more superficial waters, as already observed by Travassos et al. (1999). The inferior limit of the TSW was found around 80 meters in both periods. For the period of minimal precipitation this value is considerably shallower than the one observed by Medeiros et al. (2009a) around the ASPSP (105 meters).

\section{VERTICAL DISTRIBUTION OF THE CONSIDERED ASSEMBLAGES}

Several studies focusing on different planktonic assemblages in the area around the ASPSP were realized (e.g. Macedo-Soares et al. 2012, Melo et al. 2012, 2014, Queiroz et al. 2015). These studies focused mainly in phytoplanktonic and meso- and macrozooplanktonic assemblages. The ecology of microplanktonic heterotrophic protists such as ciliates, radiolarians and foraminifers remain poorly known especially in the tropical latitudes of the South Atlantic Ocean.

The low density of individuals per liter observed for the protistan assemblages considered in this study is characteristic of oligotrophic ecosystems. The dinoflagellates were the most abundant group. These organisms have the competitive advantage of migrating vertically in the water column so that they can access deeper waters richer in nutrients, store it within the cell, and move upward to the sunlit surface waters where photosynthesis is possible (Ji and Franks 2007). Although the determination of the trophic mode of the identified dinoflagellate species was not possible during the examination of the samples, we do know from the literature that several of the identified genus are mixotrophs, e.g. Dinophysis (Jacobson and Andersen 1994), Tripos (Smalley et al. 2003), Ornithocercus, Histioneis, Amphisolenia and Triposolenia (Tarangkoon et al. 2010), and that mixotrophy is highly important in oligotrophic regions (Tarangkoon et al. 2010, Stoecker et al. 2017). Thus, it would be expected for these organisms to present an uneven vertical distribution of abundance, which was indeed observed. The dinoflagellates exhibited higher densities at 20 meters. This depth was, in general, associated with the beginning of the thermocline and thus a density gradient. According to Blasco (1978) even a weak density gradient could lead to a concentration of the abundance of dinoflagellates on certain depths by limiting the maximum distance that they would be able to swim. The increase in the density from the base of the surface mixed layer at the depth of the onset of the thermocline could then be acting as a barrier to the dispersal of the dinoflagellates in the water column.

Foraminifers, radiolarians, aloricate and tintinnid ciliates are naturally less abundant than 
primary producers. The foraminifers are known to be more abundant in the deep chlorophyll maximum layer (DCM), which constitutes a major food source very well exploited by the planktonic foraminifera (Fairbanks and Wiebe 1980, Fairbanks et al. 1982). Although it was not possible to measure the chlorophyll content in the water column, previous works have demonstrated that the chlorophyll content is higher in deeper waters at the base of the euphotic zone in the ASPSP (Bröckel and Meyerhöfer 1999, Souza et al. 2013). Cordeiro et al. (2013) found increasing concentrations of chlorophyll with increasing depth of the water column from $\sim 25$ meters to the DCM layers between the depths of 50 and 100 meters in the waters around oceanic islands of the Equatorial Western Atlantic Ocean. If this picture remains the same for the ASPSP, the higher abundances of the foraminifers between the depths of 20 and 100 meters could be explained by the higher concentration of chlorophyll under the surface mixed layer, increasing until a DCM at the base of the euphotic zone.

The higher density values found for tintinnid ciliates at surface is consistent with the fact that, although these organisms have a broad vertical span in the oceans - with some species occurring below 600 m (Kršinić 1982) -, they are typically surface-dwelling planktonic organisms, as also observed by Thompson et al. (1999). The observed density values are, however, lower than the average 25 ind. $\mathrm{L}^{-1}$ observed by Dolan (2000) in the Mediterranean Sea, and closer to the average 11.5 ind. $\mathrm{L}^{-1}$ observed in the South Atlantic by Thompson et al. (1999). In the tropical SE Pacific Ocean, Dolan et al. (2007) observed higher mean tintinnid abundances ranging from 2-42 ind. $\mathrm{L}^{-1}$. Unfortunately we were not able to obtain cholorophyll- $a$ estimates but available data for the NW Tropical South Atlantic suggest that the chl- $a$ concentrations range from $0.05-0.87 \mu \mathrm{g} . \mathrm{L}^{-1}$ in the first 100 meters of the water column (Cordeiro et al. 2013, Jales et al. 2015), which are considerably lower than the other cited areas. This suggests that the highly oligotrophic nature of the waters around the ASPSP restrain the development of the tintinnid assemblage and keeps the abundance values low.

The radiolarians are not well studied in the Atlantic Ocean, with just a few published studies (e.g. Dworetzky and Morley 1987, Thompson et al. 1999). Our density values were close to the ones found by Thompson et al. (1999) in the South Atlantic Ocean. The slight increase in density at $100 \mathrm{~m}$ closely matches the depth of maximum density observed by some authors for colonial and polycystine radiolarians in the Pacific Ocean, around 85-100 m (Dennet et al. 2002, Zasko and Rusanov 2005).

THE PLANKTONIC ASSEMBLAGES AND THE SML AND SSL LAYERS

The stratification of the water column exerted an important influence in the dinoflagellates and tintinnid ciliates assemblages, as illustrated by the PCoA ordination diagrams. The differences among both layers are clear in terms of species composition, diversity, and richness.

The indicator value analysis revealed 27 indicator species for the SML and SSL. From these, six dinoflagellate species may be considered good indicators of the surface mixed layer, namely Tripos declinatus, $T$. teres, T. pentagonus, Histioneis milneri, Protoperidinium curtipes, and Corythodinium tesselatum. Half of these species belong to the genus Tripos. Species within this genus have historical importance as ecological indicators (Frost and Wilson 1938, Graham 1941), and have recently been suggested as a potential candidate of ecological indicator of ocean warming (Tunin-Ley and Lemée 2013), mainly because of its ubiquity and relative ease of identification. Queiroz et al. (2015) found T. declinatus and Histioneis milneri only in plankton net vertical hauls performed from 75-105 meters to the surface, and thus the only 
information available for the vertical distribution of these species in the ASPSP is that they occur in the first 100 meters. T. teres was already found exclusively in surface, $T$. pentagonus in surface, 50 meters' depth, and in the DCM, and Corythodinium tesselatum in surface and 50 meters' depth in the ASPSP (Queiroz et al. 2015). It is important to highlight that these authors collected samples at a greater distance from the archipelago and thus found the thermocline deeper than the depths reported here.

In the vertical distribution of $T$. declinatus and T. teres presented in Figure 5 it is very clear that these species present the highest values of density within the SML, and low values (if present at all) within the SSL. The fact that we have found these species with higher density values almost exclusively within the SML does not mean, however, that they do not occur in deeper layers. Baek et al. (2009) demonstrated that different species of the genus Tripos may perform diel vertical migration (DVM) in different degrees. The vertical distribution of a species is influenced by its DVM pattern. Some authors have already observed that DVM in dinoflagellates and other phytoplanktonic organisms is associated with a circadian rhythm in which the cells usually move downwards to deeper waters with higher nutrient availability during dark periods and upwards to the sunlit surface layer during light periods (Eppley et al. 1968, Baek et al. 2009). However, as stated by Blasco (1978), a minimal density gradient is sufficient to limit the vertical dispersion of dinoflagellates. The shallower depth of onset of the thermocline and associated pycnocline around the ASPSP may function as a barrier to the vertical dispersion of the dinoflagellates. Hence, our observations on different dinoflagellate species being ecological indicators of the SML and SSL are valid.

The best indicators of the SSL were the tintinnid ciliates Dictyocysta lepida, D. mitra and a non-identified Dictyocysta species. The vertical distribution of the Dictyocysta species shows clearly that $D$. lepida presented higher density values between 50-75 m, while D. mitra presented peak densities between 75-100 m. The latter species was already observed as an important component of the tintinnid assemblage between the depths of 50-100 $\mathrm{m}$ in an oligotrophic oceanic region at the South Adriatic Sea (Kršinić 1998, Kršinić and Grbec 2006). In the South Atlantic, Thompson et al. (1999) found mean average depth values of 45 and 27 meters for D. lepida and D. mitra, respectively. These values are shallower than the depth interval in which we found the peak densities of both species. However, hydrographical characteristics are completely different in their area of study, with much cooler water temperatures being found around 25-50 meters. This could explain the difference in the depth of the peak densities for both species.

The dinoflagellate assemblage was richer in species and more diverse in the SML. It was expected since photoptrophy and mixotrophy are common features among dinoflagellate species (Hansen 2011), which make these organisms more abundant in the sunlit surface waters. The higher species richness found in the SSL for the tintinnid ciliates assemblage could be related to the sinking of individuals or tintinnid loricae from the SML. This artifact was already observed by Thompson et al. (1999). However, we have found a considerable proportion of the species to be specific for a determined layer. In the case of the tintinnids, for example, $35.6 \%$ of the 38 species were exclusively found in one layer. At least $30.6 \%$ of the dinoflagellates were exclusively found in one of the layers and this pattern was particularly well observed in the radiolarians assemblage, since $46.2 \%$ of the identified species occurred exclusively in one of the layers, although no evidence for an increase/decrease of species richness or diversity of the radiolarian assemblage was detected in the depth interval here considered. This evidence 
corroborates the validity of the diversity patterns observed around the ASPSP.

We conclude that the different assemblages were affected in different ways by the presence of the permanent thermocline. The dinoflagellates exhibited the most pronounced shift in the structure of the assemblage, with good ecological indicator species for the SML, as well as higher species richness and diversity in this layer. The tintinnid ciliates assemblage is richer in species in the SSL and exhibited a few good ecological indicator species for this layer. No influence in the radiolarians assemblage in the upper 100 meters' depth interval was detected, although a trend towards an increase in density with depth was observed.

\section{ACKNOWLEDGMENTS}

This work was supported by the Conselho Nacional de Desenvolvimento Científico e Tecnológico (CNPq - grant number 405499/2012-4). We would like to thank the Coordenação de Aperfeiçoamento de Pessoal de Nível Superior (CAPES) for the PhD scholarship provided to AESFC, and to the $\mathrm{CNPq}$ for the PhD scholarship provided to JRS. We also thank the Brazilian Navy, the Comissão Interministerial para Recursos do Mar (CIRM), and the crew of the "Transmar III" ship for the logistic support. Special thanks to the biologists, which cordially assisted us in the field (Aislan G. Cunha, Renata P. S. Campelo, Patrícia S. B. Dantas, and Rafael S. Dantas) and in the logistics (Dr. Ralf Schwamborn, Dr. Manuel J. Flores-Montes, and Dr. Felipe Gaspar).

\section{REFERENCES}

ANDERSON MJ. 2001. A new method for non-parametric multivariate analysis of variance. Austral Ecol 26: 32-46.

AZAM F, FENCHEL T, FIELD JG, GRAY JS, MEYER-REIL LA AND THINGSTAD F. 1983. The ecological role of water-column microbes in the sea. Mar Ecol Prog Ser 10: 257-263.

BAEK SH, SHIMODE S, SHIN K, HAN M-S AND KIKUCHI T. 2009. Growth of dinoflagellates, Ceratium furca and
Ceratium fusus in Sagami Bay, Japan: the role of vertical migration and cell division. Harmful Algae 8: 843-856.

BALECH E. 1988. Los Dinoflagelados del Atlantico Sudoccidental. Publ Espec Inst Espan Ocenogr 1: 1-310.

BLASCO D. 1978. Observations on diel migration of marine dinoflagellates off the Baja California Coast. Mar Biol 46: 41-47.

BOLTOVSKOY D. 1999. South Atlantic Zooplankton. Leiden: Backhuys Publishers 1: 1-868.

BOLTOVSKOY D, OBERHÄNSLI H AND WEFER G. 1996. Radiolarian assemblages in the eastern tropical Atlantic: patterns in the plankton and in sediment trap samples. J Mar Sist 8: 31-51.

BRÖCKEL K AND MEYERHÖFER M. 1999. Impact of the rocks of São Pedro e São Paulo upon the quantity and quality of suspended particulate organic matter. Arch Fish Mar Res 47: 223-238.

CALBET A. 2008. The trophic roles of microzooplankton in marine systems. ICES J Mar Sci 65: 325-331.

CAMPOS TFC, BEZERRA FHR, SRIVASTAVA NK, VIEIRA MM AND VITA-FINZI C. 2010. Holocene tectonic uplift of the St Peter and St Paul rocks (Equatorial Atlantic) consistent with emplacement by extrusion. Mar Geol 271: 177-186.

CORDEIRO TA, BRANDINI FP, ROSA RS AND SASSI R. 2013. Deep chlorophyll maximum in Western Equatorial Atlantic - How does it interact with island slopes and seamounts? Marine Science 3: 30-37.

CULLEN JJ. 1982. The deep chlorophyll maximum: comparing vertical profiles of chlorophyll- $a$. Can J Fish Aquat Sci 39: 791-803.

DENNET MR, CARON DA, MICHAELS AF, GALLAGER SM AND DAVIS CS. 2002. Video plankton recorder reveals high abundances of colonial Radiolaria in surface waters of the central North Pacific. J Plankton Res 24: 797-805.

DOLAN JR. 2000. Tintinnid ciliate diversity in the Mediterranean Sea: longitudinal patterns related to water column structure in late spring-early summer. Aquat Microb Ecol 22: 69-78.

DOLAN JR, RITCHIE ME AND RAS J. 2007. The "neutral" community structure of planktonic herbivores, tintinnid ciliates of the microzooplankton, across the SE Tropical Pacific Ocean. Biogeosciences Discuss 4: 461-593.

DUFRÊNE MAND LEGENDRE P. 1997. Species assemblages and indicator species: the need for a flexible asymmetrical approach. Ecol Monogr 67: 345-366.

DWORETZKY BA AND MORLEY JJ. 1987. Vertical distribution of Radiolaria in the eastern equatorial Atlantic: analysis of a multiple series of closely-spaced plankton tows. Mar Micropal 12: 1-19. 
EPPLEY RW, HOLM-HANSEN O AND STRICKLAND JDH. 1968. Some observations on the vertical migration of dinoflagellates. J Phycol 4: 333-340.

FAIRBANKS RG, SVERDLOVE M, FREE R, WIEBE PH AND BÉ AWH. 1982. Vertical distribution and isotopic fractionation of living planktonic foraminifera from the Panama Basin. Nature 298: 841-844.

FAIRBANKS RG AND WIEBE PH. 1980. Foraminifera and chlorophyll maximum: vertical distribution, seasonal succession, and paleoceanographic significance. Science 209: 1524-1526.

FROST N AND WILSON AM. 1938. The genus Ceratium and its use as an indicator of hydrographic conditions in the Newfoundland waters. Newfoundland Res Bull 5: 1-15.

GRAHAM HW. 1941. An oceanographic consideration of the dinoflagellate genus Ceratium. Ecol Monogr 11: 99-116.

HAECKEL E. 1887. Report on the Radiolaria collected by H.M.S. Challenger. Scientific results of the voyage of H.M.S. Challenger during the years 1873-1876. Zoology, Volume XVIII. First Part.

HANSEN PJ. 2011. The role of photosynthesis and food uptake for the growth of marine mixotrophic dinoflagellates. $\mathrm{J}$ Eukaryotic Microbiol 58: 203-214.

HERBLAND A, LE BOUTEILLER A AND RAIMBAULT P. 1985. Size structure of phytoplankton biomass in the equatorial Atlantic Ocean. Deep Sea Res 32 (Part I): 819836.

HERBLAND A AND VOITURIEZ B. 1979. Hydrological structure analysis for estimating the primary production in the tropical Atlantic Ocean. J Mar Res 37: 87-101.

JACOBSON DM AND ANDERSEN RA. 1994. The discovery of mixotrophy in photosynthetic species of Dinophysis (Dinophyceae): light and electron microscopical observations of food vacuoles in Dinophysis acuminata, D. norvegica and two heterotrophic dinophysoid dinoflagellates. Phycologia 33: 97-110.

JALES MC, FEITOSA FAN, KOENING ML, MONTES MJF, ARAÚJO FILHO MC AND SILVA RA. 2015. Phytoplankton biomass dynamics and environmental variables around the Rocas Atoll Biological Reserve, South Atlantic. Braz J Oceanogr 63: 443-454.

JI R AND FRANKS PJS. 2007. Vertical migration of dinoflagellates: model analysis of strategies, growth, and vertical distribution patterns. Mar Ecol Prog Ser 344: 4961.

KOFOID CA AND CAMPBELL AS. 1929. A Conspectus of marine and fresh-water Ciliata belonging to the suborder Tintinnoinea, with descriptions of new species principally from the Agassiz expedition to the eastern Tropical Pacific 1904-1905. Univ Calif Publ Zool 34: 1-403.

KRŠINIĆ F. 1982. On vertical distribution of tintinnines (Ciliata, Oligotrichida, Tintinnina) in the open waters of the South Adriatic. Mar Biol 68: 83-90.
KRŠINIĆ F. 1998. Vertical distribution of protozoan and microcopepod communities in the South Adriatic Pit. J Plankton Res 20: 1033-1060.

KRŠINIĆ F AND GRBEC B. 2006. Horizontal distribution of tintinnids in the open waters of the South Adriatic (Eastern Mediterranean). Sci Mar 70: 77-88.

LONGHURST AR AND HARRISON WG. 1989. The biological pump: profiles of plankton production and consumption in the upper ocean. Prog Oceanogr 22: 47123.

MACEDO-SOARES LCP, FREIRE AS AND MUELBERT JH. 2012. Small-scale spatial and temporal variability of larval fish assemblages at an isolated oceanic island. Mar Ecol Prog Ser 444: 207-222.

MAIA M ET AL. 2016. Extreme mantle uplift and exhumation along a transpressive transform fault. Nat Geosci 9: 619623.

MARSHALL SM. 1969. Protozoa: Order Tintinnida. Counseil International pour 1'Exploration de la Mer, Fiches d'Identification de Zooplancton, p. 117-127.

MEDEIROS C, ARAÚJO M, FREITAS I AND ROLLNIC M. 2009a. Massas d'água da região oeste do Atlântico tropical. In: Hazin FHV (Ed), Meteorologia e Sensoriamento Remoto, Oceanografia Física, Oceanografia Química e Oceanografia Geológica. Fortaleza: Martins e Cordeiro 1: $56-69$.

MEDEIROS C, ARAÚJO M, ROLLNIC M AND FREITAS I. 2009b. Estrutura termohalina da região Oeste do Atlântico Tropical - ZEE/NE. In: Hazin FHV (Ed), Meteorologia e Sensoriamento Remoto, Oceanografia Física, Oceanografia Química e Oceanografia Geológica. Fortaleza: Martins e Cordeiro 1: 40-55.

MELO PAMC, DIAZ XFG, MACEDO SJ AND NEUMANNLEITÃO S. 2012. Diurnal and spatial variation of the mesozooplankton community in the Saint Peter and Saint Paul Archipelago, Equatorial Atlantic. Mar Biodivers Rec 5: 1-14.

MELO PAMC, MELO JR M, MACÊDO SJ, ARAÚJO MAND NEUMANN-LEITÃO S. 2014. Copepod distribution and production in a Mid-Atlantic Ridge archipelago. An Acad Bras Cienc 86: 1678-2690.

PARTENSKY F, BLANCHOT J, LANTOINE F, NEVEUX J AND MARIE D. 1996. Vertical structure of picophytoplankton at different trophic sites of the tropical northeastern Atlantic Ocean. Deep Sea Res 43 (Part I): 1191-1213.

QUEIROZAR, MONTES MF, MELO PAMC, SILVA RAAND KOENING ML. 2015. Vertical and horizontal distribution of phytoplankton around an oceanic archipelago of the Equatorial Atlantic. Mar Biodivers Rec 8: 1-13.

R CORE TEAM. 2016. R: a language and environment for statistical computing. R Foundation for Statistical Computing, Vienna, Austria. URL https://www.R-project.org. 
SANDERS RW AND WICKHAM SA. 1993. Planktonic protozoa and metazoa: predation, food quality and population control. Mar Microb Food Webs 7: 197-223.

SHERR EB AND SHERR BF. 1994. Bacterivory and herbivory: key roles of phagotrophic protists in pelagic food webs. Microb Ecol 28: 223-235.

SMALLEY GW, COATS DW AND STOECKER DK. 2003. Feeding in the mixotrophic dinoflagellate Ceratium furca is influenced by intracellular nutrient concentrations. Mar Ecol Prog Ser 262: 137-151.

SOUZA CS, LUZ JAG, MACEDO S, FLORES-MONTES MJ AND MAFALDA JR P. 2013. Chlorophyll- $a$ and nutrient distribution around seamounts and islands of the Tropical South-Western Atlantic. Mar Freshwater Res 64: 168-184.

STOECKER DK, HANSEN PJ, CARON DAAND MITRAA. 2017. Mixotrophy in the marine plankton. Annu Rev Mar Sci 9: 311-335.

STRAMMA L AND SCHOTT F. 1999. The mean flow field of the tropical Atlantic Ocean. Deep Sea Res 46 (Part II): 279-303.

TARANGKOON W, HANSEN G AND HANSEN PJ. 2010. Spatial distribution of symbiont-bearing dinoflagellates in the Indian Ocean in relation to oceanographic regimes. Aquat Microb Ecol 58: 197-213.

THOMPSON GA, ALDER VA, BOLTOVSKOY D AND BRANDINI F. 1999. Abundance and biogeography of tintinnids (Ciliophora) and associated microzooplankton in the Southwestern Atlantic Ocean. J Plankton Res 21: 1265-1298.

TRAVASSOS P, HAZIN FHV, ZAGAGLIA JR, ADVÍNCULA R AND SCHOBER J. 1999. Thermohaline structure around seamounts and islands off North-Eastern Brazil. Arch Fish Mar Res 47: 211-222.

TUNIN-LEY AAND LEMÉE R. 2013. The genus Neoceratium (planktonic dinoflagellates) as a potential indicator of ocean warming. Microorganisms 1: 58-70.

WOOD EJF. 1963. Dinoflaglellates in the Australian region. II. Recent collections. Div Fish Oceanogr Tech Pap 14: 509-563.

ZASCO DN AND RUSANOV II. 2005. Vertical distribution of radiolarians and their role in epipelagic communities of the East Pacific Rise and the Gulf of California. Biol Bull 32: 279-287. 